\title{
On surjective second order non-linear Markov operators and associated nonlinear integral equations
}

Positivity

pp $1-15$ | Cite as

- Farrukh Mukhamedov (1) Email author (far75m@yandex.ru)

- Otabek Khakimov (1)

- Ahmad Fadillah Embong (2)

1. Department of Mathematical Sciences, and College of Science, The United Arab Emirates University, , Al Ain, Abu Dhabi, UAE

2. Department of Computational and Theoretical Sciences, Faculty of Science, International Islamic University Malaysia, , Kuantan, Malaysia

Article

First Online: 21 May 2018

Received: 21 March 2018

Accepted: 17 May 2018

- 6 Downloads

\section{Abstract}

It was known that orthogonality preserving property and surjectivity of nonlinear Markov operators, acting on finite dimensional simpleces, are equivalent. It turns out that these notions are no longer equivalent when such kind of operators are considered over on infinite dimensional spaces. In the present paper, we find necessary and sufficient condition to be equivalent of these notions, for the second order nonlinear Markov operators. To do this, we fully describe all surjective second order nonlinear Markov operators acting on infinite dimensional simplex. As an application of this result, we provided some sufficient conditions for the existence of positive solutions of nonlinear integral equations whose domain are not compact.

\section{Keywords}

Non-linear Markov operator Quadratic stochastic operator Orthogonality preserving Surjective Integral equation

\section{Mathematics Subject Classification}


46L35 46L55 46A37

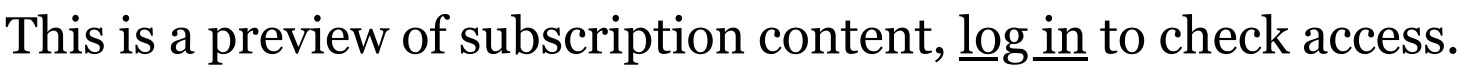

\section{Notes}

\section{Acknowledgements}

The present work is supported by the UAEU “Start-Up" Grant No. 31S259.

\section{References}

1. Akin, H., Mukhamedov, F.: Orthogonality preserving infinite dimensional quadratic stochastic operators. AIP Conf. Proc. 1676, 020008 (2015)

CrossRef (https://doi.org/10.1063/1.4930434)

Google Scholar (http://scholar.google.com/scholar_lookup?

title=Orthogonality\%2opreserving\%2oinfinite\%2odimensional\%2oquadrat ic\%20stochastic\%20operators\&author=H.\%20Akin\&author=F.\%20Mukha medov\&journal=AIP\%20Conf.\%20Proc. $\&$ volume=1676\&pages $=020008 \& p$ ublication_year $=2015$ )

2. Arutyunov, A.V.: Two problems of the theory of quadratic maps. Funct. Anal. Appl. 46, 225-227 (2012) MathSciNet (http://www.ams.org/mathscinet-getitem?mr=3075042) CrossRef (https://doi.org/10.1007/s10688-012-0028-y) MATH (http://www.emis.de/MATH-item?1275.15015) Google Scholar (http://scholar.google.com/scholar_lookup? title=Two\%20problems\%20of\%20the\%20theory\%20of\%20quadratic\%2om aps\&author $=$ AV.\%20Arutyunov\&journal=Funct.\%20Anal.\%20Appl.\&volu $\mathrm{me}=46 \&$ pages $=225-227 \&$ publication_year $=2012)$

3. Bernstein, S.N.: The solution of a mathematical problem concerning the theory of heredity. Ann. Math. Stat. 13, 53-61 (1924)

CrossRef (https://doi.org/10.1214/aoms/1177731642) Google Scholar (http://scholar.google.com/scholar_lookup? title=The\%20solution\%20of\%20a\%2omathematical\%2oproblem\%2oconce rning\%20the\%2otheory\%20of\%2oheredity\&author=SN.\%20Bernstein\&jou rnal=Ann.\%20Math.\%20Stat.\&volume $=13 \&$ pages $=53-$ 61\&publication_year=1924)

4. Ganikhodzhaev, R., Mukhamedov, F., Rozikov, U.: Quadratic stochastic operators and processes: results and open problems. Infin. Dimens. Anal. Quant. Prob. Relat. Top. 14, 279-335 (2011)

MathSciNet (http://www.ams.org/mathscinet-getitem?mr=2813492) CrossRef (https://doi.org/10.1142/So219025711004365)

MATH (http://www.emis.de/MATH-item?1242.60067)

Google Scholar (http://scholar.google.com/scholar_lookup? title=Quadratic\%20stochastic\%20operators\%20and\%2oprocesses\%3A\%20 results\%20and\%20open\%2oproblems\&author=R.\%20Ganikhodzhaev\&aut hor=F.\%2oMukhamedov\&author=U.\%2oRozikov\&journal=Infin.\%2oDime ns.\%20Anal.\%20Quant.\%20Prob.\%20Relat.\%20Top.\&volume=14\&pages= 279-335\&publication_year=2011) 
5. Jamilov, U.U.: Quadratic stochastic operators corresponding to graphs.

Lobachevskii J. Math. 34, 148-151 (2013)

MathSciNet (http://www.ams.org/mathscinet-getitem?mr=3070159)

CrossRef (https://doi.org/10.1134/S1995080213020042)

MATH (http://www.emis.de/MATH-item?1273.47096)

Google Scholar (http://scholar.google.com/scholar_lookup?

title=Quadratic\%20stochastic\%20operators\%20corresponding\%20to\%20g raphs\&author=UU.\%20Jamilov\&journal=Lobachevskii\%2OJ.\%20Math.\&v olume $=34 \&$ pages $=148-151 \&$ publication_year $=2013$ )

6. Kolokoltsov, V.N.: Nonlinear Markov Processes and Kinetic Equations.

Cambridge Univ. Press, New York (2010)

CrossRef (https://doi.org/10.1017/CBO9780511760303)

MATH (http://www.emis.de/MATH-item?1222.60003)

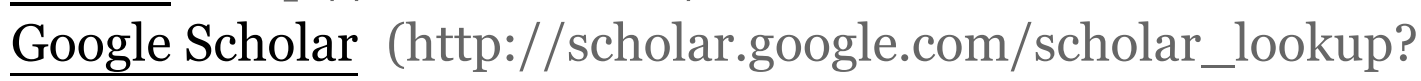

title=Nonlinear\%20Markov\%2oProcesses\%20and\%2oKinetic\%20Equation s\&author=VN.\%20Kolokoltsov\&publication_year=2010)

7. Li, C.-K., Zhang, S.: Stationary probability vectors of higher-order Markov chains. Linear Algebra Appl. 473, 114-125 (2015)

MathSciNet (http://www.ams.org/mathscinet-getitem? $\mathrm{mr}=3338328$ )

CrossRef (https://doi.org/10.1016/j.laa.2014.03.043)

MATH (http://www.emis.de/MATH-item?06433888)

Google Scholar (http://scholar.google.com/scholar_lookup?

title=Stationary\%2oprobability\%20vectors\%20of\%2ohigher-

order\%2oMarkov\%2ochains\&author=C-

K.\%20Li\&author=S.\%20Zhang\&journal=Linear\%20Algebra\%20Appl.\&vol ume $=473 \&$ pages $=114-125 \&$ publication_year $=2015$ )

8. Lyubich, YuI: Mathematical Structures in Population Genetics. Springer,

Berlin (1992)

CrossRef (https://doi.org/10.1007/978-3-642-76211-6)

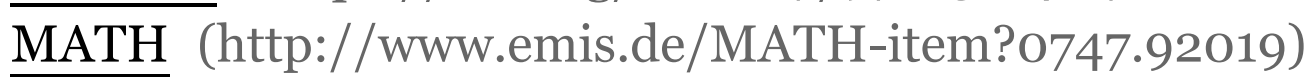

Google Scholar (http://scholar.google.com/scholar_lookup?

title=Mathematical\%20Structures\%20in\%20Population\%20Genetics\&auth or=YuI.\%20Lyubich\&publication_year=1992)

9. Mukhamedov, F.M.: On infinite dimensional Volterra operators. Russ.

Math. Surv. 55, 1161-1162 (2000)

MathSciNet (http://www.ams.org/mathscinet-getitem?mr=1840374)

CrossRef (https://doi.org/10.1070/RM2000v055no6ABEHoo0349)

MATH (http://www.emis.de/MATH-item?1010.60061)

Google Scholar (http://scholar.google.com/scholar_lookup?

title=On\%2oinfinite\%2odimensional\%20Volterra\%2ooperators\&author=F

M.\%20Mukhamedov\&journal=Russ.\%20Math.\%20Surv.\&volume=55\&pag

es=1161-1162\&publication_year=2000)

10. Mukhamedov, F., Akin, H., Temir, S.: On infinite dimensional quadratic

Volterra operators. J. Math. Anal. Appl. 310, 533-556 (2005)

MathSciNet (http://www.ams.org/mathscinet-getitem?mr=2022943)

CrossRef (https://doi.org/10.1016/j.jmaa.2005.02.022)

$\underline{\text { MATH (http://www.emis.de/MATH-item?1121.47065) }}$

Google Scholar (http://scholar.google.com/scholar_lookup?

title=On\%20infinite\%2odimensional\%20quadratic\%20Volterra\%20operat ors\&author=F.\%20Mukhamedov\&author $=$ H.\%2OAkin\&author=S.\%2OTemi 
r\&journal=J.\%2oMath.\%20Anal.\%20Appl.\&volume=310\&pages $=533-$ 556\&publication_year $=2005$ )

11. Mukhamedov, F., Embong, A.F.: On non-linear Markov operators: surjectivity vs orthogonality preserving property. Linear Multilinear Algebra. https://doi.org/10.1080/03081087.2017.1389849 (https://doi.org/10.1080/03081087.2017.1389849)

12. Mukhamedov, F., Embong, A.F., Rosli, A.: Orthogonality preserving and surjective cubic stochastic operators. Ann Funct. Anal. 8, 490-501 (2017) MathSciNet (http://www.ams.org/mathscinet-getitem? $\mathrm{mr}=3717171$ ) CrossRef (https://doi.org/10.1215/20088752-2017-0013)

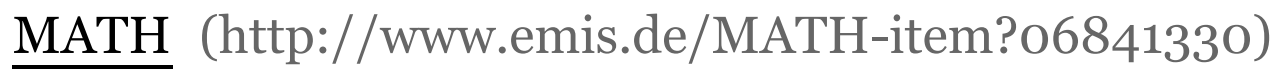
Google Scholar (http://scholar.google.com/scholar_lookup? title=Orthogonality\%2opreserving\%20and\%20surjective\%20cubic\%20stoc hastic\%20operators\&author=F.\%20Mukhamedov\&author=AF.\%20Embon g\&author=A.\%20Rosli\&journal=Ann\%20Funct.\%20Anal.\&volume=8\&page $\mathrm{S}=490-501 \&$ publication_year $=2017)$

13. Mukhamedov, F., Ganikhodjaev, N.: Quantum Quadratic Operators and Processes. Springer, Berlin (2015)

CrossRef (https://doi.org/10.1007/978-3-319-22837-2)

MATH (http://www.emis.de/MATH-item?1335.60002) Google Scholar (http://scholar.google.com/scholar_lookup? title=Quantum\%20Quadratic\%20Operators\%20and\%20Processes\&author =F.\%20Mukhamedov\&author=N.\%20Ganikhodjaev\&publication_year=201 5)

14. Mukhamedov, F., Taha, M.H.: On Volterra and orthoganality preserving quadratic stochastic operators. Miskloc Math. Notes 17, 457-470 (2016) CrossRef (https://doi.org/10.18514/MMN.2016.1090)

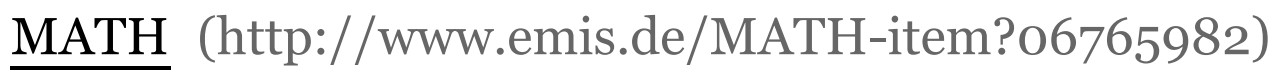

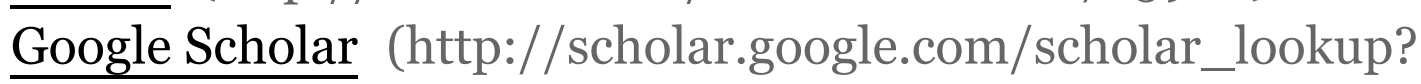
title=On\%20Volterra\%20and\%20orthoganality\%2opreserving\%2oquadrati c\%20stochastic\%20operators\&author=F.\%20Mukhamedov\&author=MH.\% 20Taha\&journal=Miskloc\%20Math.\%20Notes\&volume $=17 \&$ pages $=457-$ 470\&publication_year=2016)

15. Qi, L.: Eigenvalues of a real supersymmetric tensor. J. Symb. Comput. 40, 1302-1324 (2005)

MathSciNet (http://www.ams.org/mathscinet-getitem?mr=2178089) CrossRef (https://doi.org/10.1016/j.jsc.2005.05.007)

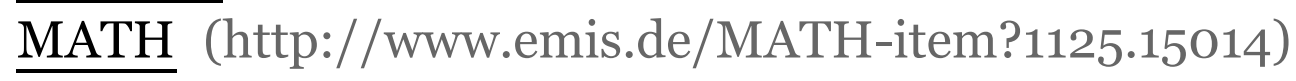
Google Scholar (http://scholar.google.com/scholar_lookup? title=Eigenvalues\%20of\%20a\%20real\%20supersymmetric\%2otensor\&auth or=L.\%20Qi\&journal=J.\%20Symb.\%20Comput.\&volume=40\&pages $=1302$ -1324\&publication_year=2005)

16. Raftery, A.: A model of high-order Markov chains. J. R. Stat. Soc. 47, 528539 (1985)

MathSciNet (http://www.ams.org/mathscinet-getitem?mr=844484)

MATH (http://www.emis.de/MATH-item?0593.62091)

Google Scholar (http://scholar.google.com/scholar_lookup?

title=A\%20model\%20of\%2ohighorder\%20Markov\%20chains\&author=A.\%20Raftery\&journal=J.\%20R.\%20 Stat.\%20Soc.\&volume $=47$ \&pages $=528-539 \&$ publication_year $=1985$ ) 
17. Saburov, M.: On the surjectivity of quadratic stochastic operators acting on the simplex. Math. Notes 99, 623-627 (2016)

MathSciNet (http://www.ams.org/mathscinet-getitem?mr=3507430)

CrossRef (https://doi.org/10.1134/Soo01434616030391)

MATH (http://www.emis.de/MATH-item?06640403)

Google Scholar (http://scholar.google.com/scholar_lookup?

title=On\%20the\%20surjectivity\%20of\%20quadratic\%20stochastic\%20oper ators\%20acting\%20on\%20the\%20simplex\&author=M.\%20Saburov\&journ

al=Math.\%20Notes\&volume=99\&pages=623-627\&publication_year=2016)

\section{Copyright information}

(C) Springer International Publishing AG, part of Springer Nature 2018

\section{About this article}

Cite this article as:

Mukhamedov, F., Khakimov, O. \& Embong, A.F. Positivity (2018). https://doi.org/10.1007/s11117-0180587-0

- DOI (Digital Object Identifier) https://doi.org/10.1007/s11117-018-0587-0

- Publisher Name Springer International Publishing

- Print ISSN 1385-1292

- Online ISSN 1572-9281

- About this journal

- Reprints and Permissions

\section{Personalised recommendations}


1. The classification of hunger behaviour of Lates Calcarifer through the integration of image processing technique and k-Nearest Neighbour learning

Taha, Z... Mukai, Y

IOP Conference Series: Materials Science and Engineering (2018)

2. The Identification of Hunger Behaviour of Lates Calcarifer through the Integration of Image Processing Technique and Support Vector Machine Taha, Z... Mukai, Y.

IOP Conference Series: Materials Science and Engineering (2018)

3. Establishing Relationship between Process Parameters and Temperature during High Speed End Milling of Soda Lime Glass

Bagum, Mst. Nasima... Ali, Mohammad Yeakub

IOP Conference Series: Materials Science and Engineering (2018)

Want recommendations via email? Sign up now

Powered by: Recommended

\section{SPRINGER NATURE}

(C) 2017 Springer Nature Switzerland AG. Part of $\underline{\text { Springer Nature. }}$

Not logged in International Islamic University Malaysia (IIUM) (2000621865) - 4972 SpringerLink Malaysia eBook Consortium-2009-2010 copyright (3000134874) - 6816 SpringerLink Malaysia eJournal Consortium Higher Education (3000155375) - 8354 Springerlink Malaysia consortium (3000519906) - 10122 SpringerLink Malaysia eJourna Consortium - Higher Education (3000716851) - SpringerLink Malaysia eJournal Consortium - Higher Education (3000916360) 210.48.222.9 